\title{
Method for Distinguishing the Digital Images in Different Formats
}

\author{
Kobozeva A.A., Bobok I.I., Kushnirenko N.I. \\ Odessa Polytechnic National University \\ Odesa, Ukraine
}

\begin{abstract}
Nowadays, the energy systems are considered to be the main vital factor for the functioning of society. Today, this part of the infrastructure cannot exist without the informative infrastructure, so it needs efficient information and cyber protection. To detect the data integrity violations is one of the main tasks in the field of information security. In the paper, the digital images are considered as information content. The image integrity violation often results in the change of the original storage format. The format change is an indicator of the image transformation. At the same time, this fact can be considered as an indirect indicator of its integrity violation. The effective method for distinguishing the original image in lossless format from the image re-saved to lossless format from lossy format is developed in the current work. The proposed method is an improvement of the method developed by the authors earlier. The method is based on the analysis of the number of non-intersecting image blocks, in which the normalized gap of maximum singular value increases due to the image's re-saving into a lossy format. The algorithmic implementation of the improved method allowed us to reduce the examination time in 38-56 times for more than 50\% of images originally stored in lossy format compared to the prototype method. The algorithm efficiency was evaluated using Type I and Type II errors, as well as applying the accuracy coefficient (ACC). As a result of testing, Type I error was not detected, Type II error rate was 3.6\%, ACC $=0.98$. The obtained estimations are independent of the quality factor used when the image was initially saved in a lossy format. The developed algorithm is more efficient than almost all modern analogues, except for the prototype one. The theoretical basis of the proposed improved method makes it possible to use it for the study of the digital image integrity, violated by a minor disturbance, in particular for steganalysis in conditions of low capacity of the hidden communication channel.
\end{abstract}

Keywords: integrity violation, digital images, lossy format, lossless format, singular value.

DOI: https://doi.org/10.52254/1857-0070.2022.1-53.09

UDC: 004.056

Metodă pentru separarea imaginilor digitale în diferite formate de stocare

Kobozeva A.A., Boboc I.I., Kușnirenco N.I.

Universitatea națională «Odesika Politehnica», Odesa, Ucraina

Rezumat. Rezultatul abaterilor de la integritatea imaginii digitale este adesea schimbarea formatului de stocare a acesteia, care este considerat un indicator indirect al convertirii sale și atribuie actualitate sarcinii face actă sarcina de separare a imaginii în diferite formate de stocare, scoțând la evidență faptul că imaginea a fost resalvată. Una din cele mai eficiente metodele moderne, ce soluționează această problemă se bazează pe analiza funcției $y(Q F)$ care reflectă dependența numărului de blocuri ale imaginii, în care este majorată separația normată a numărului maxim singular la re-salvarea în format cu pierderi, de coeficientul calități $Q F$. Scopul acestei lucrări este de a reduce investițiile de timp pentru separarea imaginii digitale originale în format fără pierderi de cea care a fost re-salvată în format fără pierderi din formatul cu pierderi, păstrând în același timp o eficiență ridicată a separării. Scopul este atins printr-o alegere rezonabilă a unui număr mic de factori de calitate dintre toți factorii de calitate posibili pentru formatul cu pierderi în cauză, care determină proprietățile caracteristice ale funcției $\mathrm{y}(\mathrm{QF})$ independent de specificitatea formatului. Cel mai important rezultat al acestei lucrări este elaborarea metodei și implementarea sa algoritmică a acesteia, care a permis reducerea de 38-56 ori a timpului de examinare pentru mai mult de 50\% din imaginile stocate iniţial într-un format cu pierderi în comparație cu metoda prototip, astfel eficiența algoritmului sporește eficiența analogiile: erori de gradul $1 \mathrm{nu}$ au fost identificate, erori de gradul 2 au constituit 3.6\%. Semnificația rezultatelor obținute constă în asigurarea posibilității examinării eficiente a imaginii, indiferent de factorul de calitate al stocării sale inițiale într-un format cu pierderi în regim de timp real.

Cuvinte-cheie: încălcare a integrităţii, imagine digitală, format cu pierderi, format fără pierderi, separare normalizată a numărului maxim singular.

(С Кобозева А.А., Бобок И.И.,

Кушниренко Н.И., 2022 


\section{Метод отделения цифровых изображений в разных форматах хранения \\ Кобозева А.А., Бобок И.И., Кушниренко Н.И.}

Национальный университет «Одесская Политехника», Одесса, Украина

Аннотация. Результатом нарушения целостности цифрового изображения часто является изменение формата его хранения, что рассматривается как косвенный показатель его преобразования и делает актуальной задачу отделения изображений в разных форматах хранения, выявления факта пересохранения изображения. Один из наиболее эффективных современных методов, решающих такую задачу, основан на анализе функции $y(Q F)$, которая отражает зависимость количества блоков изображения, в которых увеличивается нормированная отделенность максимального сингулярного числа при пересохранении в формат с потерями, от коэффициента качества $Q F$. Значимым недостатком соответствующего алгоритма является его значительная вычислительная сложность при экспертизе любого изображения. Целью работы является сокращение временных затрат для отделения оригинального цифрового изображения в формате без потерь от такого, которое было пересохранено в формат без потерь из формата с потерями, с сохранением высокой эффективности отделения. Цель работы достигнута за счет обоснованного выбора незначительного количества коэффициентов качества среди всех возможных для рассматриваемого формата с потерями, которые определяют характерные свойства функции $y(Q F)$ независимо от конкретики формата. Наиболее важным результатом работы является разработка метода и его алгоритмической реализации, которая позволила уменьшить в 38-56 раз время экспертизы для более чем 50\% изображений, первоначально хранящихся в формате с потерями, по сравнению с методом-прототипом, при этом эффективность алгоритма превышает эффективность аналогов: ошибки 1-го рода выявлены не были, ошибки 2-го рода составили 3.6\%. Значимость полученных результатов заключается в обеспечении возможности эффективной экспертизы изображения независимо от коэффициента качества его первичного сохранения в формате с потерями в режиме реального времени. Преимуществом предложенного метода является обеспеченная возможность его использования при экспертизе целостности цифрового изображения в условиях малых возмущающих воздействий.

Ключевые слова: нарушение целостности, цифровое изображение, формат с потерями, формат без потерь, нормированная отделенность максимального сингулярного числа.

\section{I. ВВЕДЕНИЕ}

Энергетические системы являются одними из основных жизненно необходимых для функционирования современного общества. На сегодняшний день эта часть инфраструктуры невозможна без инфраструктуры информационной, а потому нуждается в эффективной информационной и киберзащите.

Целостность информации - один из критериев ее защищённости [1-5], а выявление нарушения целостности - одна из основных задач пассивной защиты информации [6,7]. Нарушение целостности может происходить разными способами и иметь разные цели, но всегда приводит к неоригинальному контенту, нуждаясь в эффективных методах выявления [8-11]. Далее, как информационный контент в работе рассматриваются цифровые изображения (ЦИ). Результатом нарушения целостности ЦИ часто является изменение формата (c/без потерь) его хранения относительно первоначального. Так, например, при организации скрытого канала связи [12-13] с использованием стеганографических методов, которые не являются устойчивыми к атаке сжатием [14], в частности LSB-метода [15-16], изображение-стеганосообщение сохраняется в формате без потерь (ФбП). Если ЦИ-контейнер при этом был в формате с потерями (ФсП), то для него, вместе с нарушение целостности в результате встраивания дополнительной информации, происходит изменение формата. Таким образом, выявление факта изменения формата цифрового контента, в частности ЦИ, является показателем его преобразования, косвенным показателем на нарушение его целостности, и, как следствие, актуальной задачей в области информационной безопасности.

Учитывая то, что наиболее распространенными на сегодня при создании, хранении, пересылке ЦИ являются ФсП [17], конкретизируем задачу следующим образом: отделить оригинальное ЦИ в ФбП от такого, которое было пересохранено в ФбП из ФсП. Решение этой задачи может использоваться для разных целей, в частности оно будет полезным для стеганоанализа [18-22] в условиях малой пропускной способности скрытого канала связи $[14,23]$. И хотя этой задаче уделяется внимание в современных 
научных публикациях, но это внимание является недостаточным. Основной причиной этого, по мнению авторов настоящей статьи, является пренебрежение исследователями критериев защищенности информации косвенными признакам в пользу прямых. Конечно, установление явных сигнатур, например, для стеганосообщения, полученного LSB-методом [14], является предпочтительным, по сравнению с выявлением факта пересохранения ЦИ без явного понимания причины этого пересохранения. Однако в современных условиях развития информационных технологий неавторизованные изменения ЦИ, в частности, при организации стеганографического канала связи, могут быть настолько незначительными, что их выявление будет проблематичным, вплоть до невозможного, с использованием любого (визуального, статистического, сигнатурного) [14] стеганоаналитического метода. В такой ситуации выявление наличия пересохранения ЦИ может оказаться единственным возможным указателем на неавторизованные изменения контента. В связи с этим актуальным требованием для любого метода отделения ЦИ в разных форматах хранения является их работоспособность в условиях дополнительных (незначительных) возмущений контента, что далеко не всегда имеет место для существующих методов, но обеспечивается предложенным в настоящей работе.

Главным недостатком метода, разработанного в [24], является его неприемлемо низкая эффективность в условиях, когда значение коэффициента качества (КК) $Q F$, который использовался при получении оригинального ЦИ в формате JPEG, больше 60. Эффективность здесь оценивается с помощью значения точности (ACC) [25]:

$$
A C C=(T P+T N) /(T P+F N+T N+F P),(1)
$$

где ТP (True Positive) - число правильно выявленных пересохраненных из ФсП в ФбП ЦИ (истинно-положительный результат); ТN (True Negative) - число правильно выявленных оригинальных ЦИ в ФбП (истинно-отрицательный результат); FP (False Positive) - число оригинальных ЦИ в ФбП, ошибочно принятых за пересохраненные в ФбП из ФсП (ложно-положительный результат (ложная тревога) или ошибка II рода); FN (False Negative) - число ЦИ, пересохраненных в ФбП из ФсП, ошибочно признанных оригинальными в ФбП (ложноотрицательный результат или ошибка I рода). Так для $\mathrm{QF}=70$ значение $\mathrm{ACC}<0.8$.

Значительно более эффективным, по сравнению с предыдущим, является алгоритм, предложенный в [26], хотя для ЦИ, полученных со значительным QF, значение АСС (1) здесь падает до 0.93.

Ни один из методов [24,26] не рассчитан на работу в условиях дополнительных возмущающих воздействий, кроме того недостатком каждого из них является снижение эффективности с увеличением QF оригинального ЦИ, оставляя актуальной задачу обеспечения эффективного отделения изображений в разных форматах независимо от КК (который в реальных условиях эксперту, как правило, неизвестен), способ решения которой предлагается в настоящей статье.

В [23] разработан метод, основанный на учете последовательных цветовых триад триплетов в матрице уникальных цветов ЦИ, производящий экспертизу изображения в пространственной области. Показатели эффективности алгоритмической реализации метода: $\mathrm{ACC}=0.96$ (ошибки I рода составили $3 \%$, II рода - 4.5\%), однако КК, использованные для ЦИ в ФсП, участвовавших в вычислительном эксперименте, в работе не приведены.

В [27] предложен метод отделения оригинального ЦИ в ФбП от такого, которое было пересохранено в ФбП из ФсП, с последующим определением для последнего KK QF, использованного при первичном сжатии. Метод разработан на основе результатов исследования свойств сингулярных чисел (СНЧ) [28] блоков матрицы изображения, полученных путем стандартного разбиения, а именно свойств дискретной функции $z(Q F), \quad$ которая определяет количество блоков изображения, в которых не меняется норивмированная отделенность максимального сингулярного числа блока (НОМСЧ) при пересохранении его в ФсП с коэффициентом качества QF. Если $B-l \times l$-блок ЦИ, СНЧ которого $\sigma_{1} \geq \sigma_{2} \geq \ldots \geq \sigma_{l} \geq 0$ [29], то нормированная отделенность сингулярного числа $\sigma_{i}$ блока $B$ определяется следующим образом [27]: 


$$
\operatorname{svdgap}_{n}(i)=\min _{i \neq j}\left|\bar{\sigma}_{j}-\bar{\sigma}_{i}\right|
$$

где $\bar{\sigma}_{i}, i=1, \ldots, l,-$ элементы нормированного вектора $\mathrm{CHЧ} \bar{\sigma}=\frac{\left(\sigma_{1}, \sigma_{2}, \ldots, \sigma_{l}\right)^{T}}{\left\|\left(\sigma_{1}, \sigma_{2}, \ldots, \sigma_{l}\right)\right\|}, \quad\|\cdot\| \quad-$ векторная норма. Нормированная отделенность максимального СНЧ блока определяется в соответствии с (2):

$$
\operatorname{svdgap}_{n}(1)=\bar{\sigma}_{1}-\bar{\sigma}_{2}
$$

В [27] приведены две алгоритмические реализации метода, которые отличаются способом определения выраженного локального максимума функции $z(Q F)$. Для алгоритмической реализации, где как выраженный рассматривался локальный максимум, наибольший по значению, ошибки I рода составили $0.34 \%$, II рода - $8 \%$ $(\mathrm{ACC}=0.96)$; когда выраженный локальный максимум определялся по наибольшей глубине, ошибки I рода отсутствовали, но II рода составили $10 \%(\mathrm{ACC}=0.95)$. ЦИ в ФсП, которые были задействованы в эксперименте, брались с $Q F \in\{55,60,65,70,75,80,85,90,95\}$. Для предложенного метода наблюдается значительное уменьшение ошибок I рода в сравнении с предыдущими методами, но количество ошибок II рода остается значительным.

Методы, предложенные в [23,27], не рассчитаны на работу в условиях дополнительных возмущающих воздействий.

Результатом дальнейшего исследования свойств НОМСЧ (3) блока ЦИ стал метод выявления нарушения целостности ЦИ в результате незначительного возмущающего воздействия, предложенный в [30], эффективность которого превысила эффективность аналогов. Математический базис метода позволяет его применение для отделения оригинального ЦИ в ФбП от такого, которое было пересохранено в ФбП из ФсП. Экспертиза ЦИ здесь сводится к анализу свойств дискретной функции $y(Q F)$, которая определяет относительное количество блоков ЦИ, для которых возросла НОМСЧ в результате пересохранения в ФсП с КК $Q F$. Эффективность метода будет тем выше, чем более точно построена функция $y(Q F)$, то есть чем большее количество аргументов $Q F$ используется. Построение $y(Q F)$ с учетом всех возможных для этого формата коэффициентов качества (для формата JPEG $Q F \in\{1,2, \ldots, 99,100\})$ предусматривает пересохранение ЦИ со всеми возможными КК, приводит к значительной вычислительной сложности алгоритмической реализации метода, что является его недостатком, учитывая значительные объемы информационных контентов, которые подвергаются сегодня экспертизе целостности. Высокая эффективность метода обосновывает необходимость его усовершенствования для уменьшения временных затрат.

Таким образом, оценки эффективности алгоритмических реализаций рассмотренных методов, их временные характеристики остаются такими, которые могут и должны быть улучшенными. Такие улучшения являются критически необходимыми в условиях стеганоанализа, при ограниченном времени экспертизы значительного количества ЦИ, последовательных кадров цифрового видео и т.п.

Целью работы является сокращение временных затрат для отделения оригинального ЦИ в ФбП от такого, которое было пересохранено в ФбП из ФсП с сохранением высокой эффективности отделения независимо от коэффициента качества ЦИ в ФсП путем усовершенствования предложенного в [30] метода.

Для достижения поставленной цели в работе решаются следующие задачи:

1. Обосновать выбор незначительного количества КК среди всех возможных для рассматриваемого ФсП, которые определяют характерные свойства функции $y(Q F)$ независимо от конкретики ФсП;

2. Провести дополнительные теоретические и экспериментальные исследования свойств функции $y(Q F)$ для выбранных значений аргумента $Q F$, результаты которых дадут возможность для уменьшения количества пересохранений исследуемого ЦИ в ФсП при его экспертизе, оставляя высокой эффективность соответствующего метода;

3. Провести усовершенствование метода, предложенного в [30], с целью обеспечения возможности уменьшения вычислительной сложности для решения задачи отделения оригинальных ЦИ в ФбП от 
таких, которые были пересохранены в ФбП из ФсП;

4. Разработать алгоритмическую реализацию предложенного метода для конкретного ФсП; провести оценку ее эффективности, в том числе сравнительную.

\section{II. МЕТОДЫ}

Пусть формальным представлением ЦИ является $n \times m$-матрица $F$, которая разбивается стандартным образом [31] на непересекающиеся $l \times l$-блоки, произвольный из которых далее обозначается $B$. Пусть $J P$ множество всех возможных значений КК $Q F$ для ФсП, который используется при пересохранении исследуемого ЦИ в процессе экспертизы: $Q F \in J P$.

Теоретически максимальное (близкое к максимальному) значение для количества блоков исследуемого ЦИ, в которых возрастет НОМСЧ в результате пересохранения изображения в ФсП, независимо от формата (c/без потерь) исследуемого изображения, будет тогда, когда пересохранение происходит с самым малым из возможных для данного формата КК $Q F$, который далее обозначается $Q F_{\text {min }}$ (например, для JPEG $Q F_{\min }=1$ ):

$$
Q F_{\min }=\min J P .
$$

Действительно, этот вариант в целом отвечает самым большим коэффициентам в матрице квантования, используемой в процессе сжатия [31]. Он приведет к самому большому количеству обнуленных частотных коэффициентов, и, как следствие, к самому большому общему количеству СНЧ блоков, которые станут сравнимы с нулем. Таким образом, рассмотренный вариант будет соответствовать самому большому количеству блоков исходного ЦИ, где произойдет увеличение НОМСЧ в пересохраненном изображении, по сравнению с вариантом, когда пересохранение исходного ЦИ будет осуществляться с $Q F>Q F_{\min }$.

Однако на практике возможна ситуация, когда

$$
y\left(Q F_{\min }\right) \neq \max _{Q F} y(Q F) .
$$

Это происходит в силу следующего. Матрицы квантования для близких значений $Q F$ отличаются незначительно. Округления, которые обязательно происходят при работе в системе чисел с плавающей точкой, в том числе, при вычислении НОМСЧ, значение которой, как следует из (3), незначительно $\left(0<\operatorname{svdgap}_{n}(1) \leq 1\right)$, могут привести к тому, что на практике из-за накопления вычислительной погрешности $\max _{Q F} y(Q F)=y(\overline{Q F}), \quad$ где $\quad \overline{Q F} \neq Q F_{\min }, \quad$ хотя $y\left(Q F_{\min }\right) \quad$ будет близко к значению $\max _{Q F} y(Q F)$, не смотря на (5).

Значение $\min _{Q F} y(Q F)$ для ЦИ в ФсП часто будет определяться тем КК, который отвечает значению $Q F$ первичного сжатия исходного ЦИ [30]. Для исходного ЦИ в ФбП $\min _{Q F} y(Q F)$ определяется, учитывая монотонное невозрастание функции $y(Q F)$, наибольшим из возможных значений $Q F$ :

$$
Q F_{\max }=\max J P .
$$

(например, для JPEG $Q F_{\max }=100$ ), что вытекает из вышесказанного з учетом [30]. И хотя для функции $y(Q F)$ для ЦИ в ФсП нарушается монотонное невозрастание, но общая тенденция уменьшения количества блоков ЦИ, в которых увеличивается НОМСЧ при пересохранении с потерями, с увеличением КК имеет место, как следует из вышесказанного. Полученный вывод иллюстрирует рис. 1, где для наглядности приведены также графики сглаживающих функций, обозначенные $\bar{y}(Q F)$ (под соответствующими ЦИ понимаются изображения, которые отличаются лишь форматом (с/без потерь) хранения).

Таким образом,

$$
\begin{aligned}
& \max _{Q F} y(Q F)-\min _{Q F} y(Q F) \approx \\
& \approx y\left(Q F_{\text {min }}\right)-y\left(Q F_{\text {max }}\right)
\end{aligned}
$$

Ниже рассматриваются соответствующие ЦИ. Обозначим их матрицы как $F_{T}$ (для ЦИ в ФбП) и $F_{J}$ (для ЦИ в ФсП).

Функцию $y(Q F), \quad$ которая отвечает оригинальному ЦИ в ФбП, обозначим 
$y_{0}(Q F)$. Предположим, что из одного ЦИ в ФбП получены два соответствующих изображения путем пересохранения его с потерями с КК $Q F=Q F_{1}$ и $Q F=Q F_{2}$, при этом $Q F_{1}<Q F_{2}$. Соответствующие функции $y(Q F)$ для этих двух изображений обозначим $y_{1}(Q F), \quad y_{2}(Q F) . \quad$ Если $\quad Q F<Q, \quad$ то $y_{1}(Q F)<y_{2}(Q F)$, где $Q-$ пороговое значение, которое определяется экспериментально для конкретного ФсП (для формата JPEG $Q=75$ ). С геометрической точки зрения это означает, что для $Q F \in J P \cap\left[Q F_{\min }, Q\right)$ график функции $y_{1}(Q F)$ будет расположен ниже графика $y_{2}(Q F)$ вдоль оси ординат. Таким образом:

$$
y_{1}\left(Q F_{\min }\right)<y_{2}\left(Q F_{\min }\right)
$$

(связь между $y_{1}\left(Q F_{\max }\right)$ и $y_{2}\left(Q F_{\max }\right)$ может отличаться от соотношения (8) для конкретных изображений).

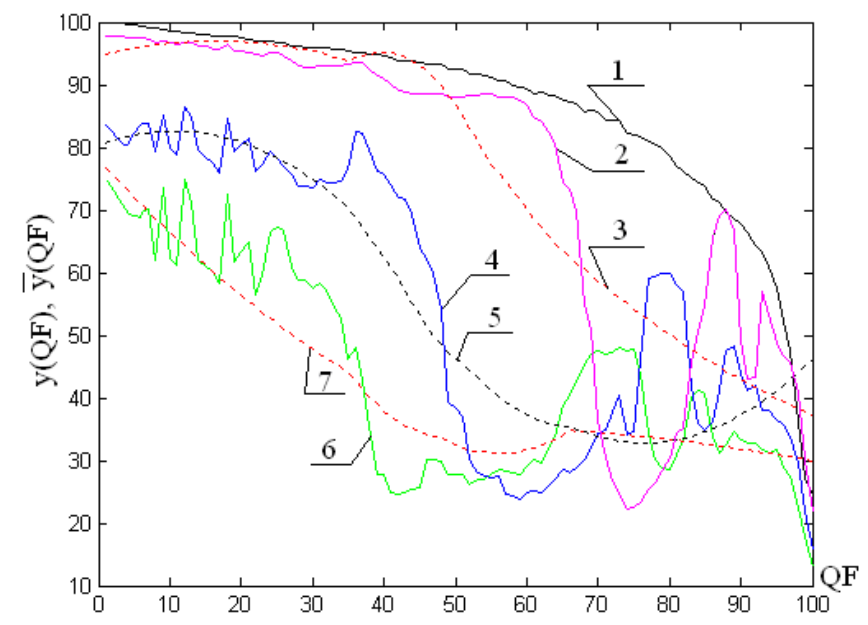

Рис. 1. Графики функций $y(Q F)$ и $\bar{y}(Q F)$ для соответствующих ЦИ:

$\mathbf{1}-y(Q F)$ для ЦИ в формате TIF; 2, 3 - y $(Q F)$ для ЦИ в формате JPEG (QF=85) и $\bar{y}(Q F)$ соответственно; 4, 5 - y $(Q F)$ для ЦИ в формате JPEG $(Q F=75)$ и $\bar{y}(Q F)$ соответственно; 6,7 -

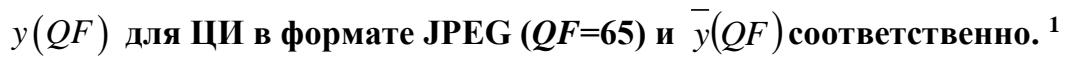

Заметим, что соотношение (8) определяет направление исследований, целью которых является обеспечение возможности определения/оценки КК оригинального ЦИ в ФсП и, как следствие, определение/оценки пропускной способности скрытого канала связи, когда как контейнер используется ЦИ в ФсП.

Соотношение (8) очевидно можно уточнить:

$$
y_{1}\left(Q F_{\text {min }}\right)<y_{2}\left(Q F_{\text {min }}\right)<y_{0}\left(Q F_{\text {min }}\right) \text {, }
$$

при этом для задачи, которая решается в работе, главной является ${ }^{1}$ Appendix 1 правая часть (9). Действительно, каким бы не был КК при получении соответствующего ЦИ в ФсП из ЦИ в ФбП, в изображении с матрицей $F_{J}$ вклад высокочастотной составляющей будет меньшим, чем у соответствующего ЦИ с матрицей $F_{T}$, что приведет к уменьшению наименьших СНЧ (увеличение НОМСЧ) блоков $F_{J}$ в сравнении с соответствующими СНЧ соответствующих блоков $F_{T}$. Поскольку в общем случае можно утверждать, что чем меньше НОМСЧ в блоке ЦИ, тем больше вероятность ее роста при пересохранении ЦИ с потерями, то, как правило,

$$
y_{0}(Q F)>y(Q F),
$$

где $y(Q F) \quad$ отвечает любому соответствующему ЦИ в ФсП на всей области определения $Q F \in J P$, т.е. 


$$
y_{0}\left(Q F_{\min }\right)>y\left(Q F_{\min }\right) .
$$

Соотношение (11) может быть использовано для отделения оригинального ЦИ в ФбП от такого, которое было пересохранено в ФбП из ФсП.

Не смотря на то, что соотношение между $y_{1}\left(Q F_{\max }\right) \quad$ и $y_{2}\left(Q F_{\max }\right)$ для конкретных изображений могут отличаться, из вытекает, что, как правило,

$$
\begin{aligned}
& \left(y_{0}\left(Q F_{\max }\right)>y_{1}\left(Q F_{\max }\right)\right) \& \\
& \&\left(y_{0}\left(Q F_{\max }\right)>y_{2}\left(Q F_{\max }\right)\right)
\end{aligned}
$$

независимо от конкретных значений $Q F_{1}$, $Q F_{2}$, при этом $y\left(Q F_{\max }\right)$ не может быть значительным при пересохранении любого ЦИ, независимо от того, в каком формате это изображение (з/без потерь) было получено первоначально, а для ЦИ в ФсП - независимо от значения КК. Действительно, каким-бы ни было исходное ЦИ, при пересохранении его с $Q F=Q F_{\max } \quad$ частотные коэффициенты его блоков в результате восстановления после квантования и округления изменяться незначительно, что в итоге приведет к тому, что количество блоков, в которых произойдет при пересохранении ЦИ с потерями увеличение НОМСЧ, будет небольшим, независимо от формата исходного ЦИ. Таким образом, хотя для $y\left(Q F_{\max }\right)$ будет иметь место (12), но сами значения $y\left(Q F_{\max }\right)$ будут незначительно отличаться друг от друга для ЦИ в разных форматах и с разными $Q F$, что подтверждается на практике результатами вычислительного эксперимента, в котором были задействованы: $450400 \times 400$-ЦИ в ФбП (TIF) (300 ЦИ из базы img_Nikon_D70s [32] (множество $M_{T i f}^{1(400)}$ ) и 150 ЦИ из базы 4cam_auth [33] (множество $\left.M_{\text {Tif }}^{2(400)}\right)$ ) $M_{\text {Tif }}^{1(400)} \cup M_{\text {Tif }}^{2(400)}, 1800$ ЦИ в ФсП, которые составили 4 множества: $\quad M_{J p e g, 70}^{1(400)} \cup M_{J p e g, 70}^{2(400)}$, $M_{\text {Jpeg, }, 75}^{1(400)} \cup M_{\text {Jpeg }, 75}^{2(400)}, \quad \quad M_{J p e g}^{1(400)} \cup\left(\cup M_{J p e g}^{2(400)}\right.$, $M_{\text {Jpeg, }, 90}^{1(400)} \cup M_{\text {Jpeg, } 90}^{2(400)}, \quad$ каждое $\quad$ из которых содержало по 450 изображений, полученных пересохранением изображений из множества $M_{\text {Tif }}^{1(400)} \cup M_{\text {Tif }}^{2(400)} \quad$ в $\quad$ формат JPEG с
$\begin{array}{llr}Q F \in\{70,75,80,90\} & \text { (наиболее } & \text { часто } \\ \text { используемые } & \text { на } & \text { практике }\end{array}$ соответственно. Так разброс для средних значений количества блоков ЦИ, в которых произошло увеличение НОМСЧ при пересохранении с потерями с $Q F=Q F_{\max }=100$, составляет от 12 до 21 (табл. 1).

Принципиально иная картина наблюдается при пересохранении ЦИ с $Q F=Q F_{\min }=1$. Разброс для средних значений рассматриваемого количества блоков здесь намного больше - от 65 до 98 (табл. 2), что является ожидаемым. Действительно, когда ЦИ пересохраняется с потерями с наименьшим из возможных коэффициентом качества, то значительные изменения в значениях частотных коэффициентов произойдут в любом ЦИ, если говорить о ЦИ, которые используются чаще всего на практике, то есть ЦИ, которые сохранены без потерь, или с потерями, но с $Q F$, обеспечивающими их качество восприятия, то есть такими, которые значительно отличаются от $Q F_{\min }$ (например, для формата JPEG - с $Q F>50)$. Значения $y\left(Q F_{\min }\right)$, их отличия будут значительно зависеть от формата оригинального изображения (c/без потерь), а для ЦИ, которое изначально было в $\Phi c П$, от $Q F$. В ЦИ в ФбП высокочастотная составляющая имеет первозданный вид, наименьшие $\mathrm{CHЧ}$ в блоках такого изображения несравнимы с нулем. Его пересохранение с $Q F=Q F_{\min }$ является первичным сжатием с потерями, которое гарантировано обнулит (на практике благодаря округлениям в системе чисел с плавающей точкой сделает сравнимыми с нулем) наименьшие СНЧ почти всех блоков матрицы изображения, и, как следствие, в результате увеличит НОМСЧ почти в каждом блоке, то есть для ЦИ без потерь $y\left(Q F_{\min }\right) \approx 100$. Если ЦИ изначально было в ФсП, то для некоторых его блоков еще до пересохранения с потерями, которое происходит в ходе экспертизы, наименьшие СНЧ уже сравнимы с нулем, поэтому повторное сжатие приведет к увеличению НОМСЧ в меньшем количестве блоков, чем в ЦИ в ФбП. Для оригинального ЦИ в ФсП с $Q F$, значительно отличающимся от $Q F_{\max }$ (например, для JPEG - $3 \quad Q F \leq 75$ ), высокочастотная составляющая уже 
значительно «пострадала» еще в оригинальном ЦИ, количество блоков, в которых еще до пересохранения с потерями наименьшие СНЧ уже сравнимы с нулем, будет значительным, поэтому будет значительным и отличие между $y\left(Q F_{\text {min }}\right)$ для ЦИ в ФбП и соответствующего ЦИ в ФсП. Для последнего, как правило, $y\left(Q F_{\min }\right)<<100$. Таким образом, соотношение (7), а именно его правая часть $y\left(Q F_{\min }\right)-y\left(Q F_{\max }\right)$, где $y\left(Q F_{\max }\right)$ мало зависит от формата, в сравнении с $y\left(Q F_{\min }\right)$, которое значительно изменяет свое значение в случае изменения формата и $Q F$ для исследуемого ЦИ, принципиально может использоваться для обеспечения возможности отделения ЦИ в ФсП от ЦИ в ФбП.

Bce вышеизложенное подтверждается результатами вычислительного эксперимента, представлеными в табл. 1,2, рис. 2-5, при этом обозначение Tif по оси абсцисс на рис. 2 соответствует ЦИ в формате без потерь - Tif.

Из построенных гистограмм видно, что учет количества блоков ЦИ, для которых увеличилась НОМСЧ при пересохранении изображения в формат JPEG с $Q F=Q F_{\max }=100, \quad$ принесет очевидный выигрыш во времени экспертизы, основанной на анализе дискретной функции $y(Q F)$, ЦИ в ФсП для наибольшего количества изображений (в сравнении с учетом $y\left(Q F_{\min }\right)$, значения $\left.y\left(Q F_{\min }\right)-y\left(Q F_{\max }\right)\right) \quad-\quad$ в совокупности больше половины ЦИ.

На рис. 4 этой части изображений отвечают части гистограмм слева от красной вертикальной прямой - это изображения, для которых $y\left(Q F_{\max }\right)<13.6$. Надо заметить, что для наиболее распространенного на практике KК $Q F=75$ (JPEG) количество таких изображений составляет почти половину от общего количества. Для экспертизы таких ЦИ их достаточно будет пересохранить в ФсП лишь один раз.

Этот факт учитывается при разработке соответствующего экспертного метода.

Приведенные результаты эксперимента показывают, что отличие между качественными и количественными характеристиками гистограмм для ЦИ в ФбП и ФсП тем меньше, чем больше КК оригинального ЦИ с потерями.

Можно предположить, что для значительных КК (например, для JPEG при $Q F>85)$ нельзя получить такие оценки для (7), (11), (12), которые бы позволили для большинства изображений эффективно отделять ЦИ в ФбП от ЦИ в ФсП без построения функции $y(Q F)$ на области $Q F \in J P$.

Таблица $1^{2}$.

Количество блоков ЦИ, в которых происходит увеличение НОМСЧ при пересохранении с потерями с $Q F=100(\%)$

\begin{tabular}{|c|c|c|c|c|c|}
\hline $\begin{array}{c}\text { Формат ЦИ } \\
\text { Image format }\end{array}$ & TIF & $\begin{array}{c}\text { JPEG } \\
(Q F=90)\end{array}$ & $\begin{array}{c}\text { JPEG } \\
(Q F=80)\end{array}$ & $\begin{array}{c}\text { JPEG } \\
(Q F=75)\end{array}$ & $\begin{array}{c}\text { JPEG } \\
(Q F=70)\end{array}$ \\
\hline $\begin{array}{c}\text { Минимальное количество } \\
\text { Міnimal number }\end{array}$ & 13.6 & 6 & 0.5 & 0.03 & 0.02 \\
\hline $\begin{array}{c}\text { Максимальное количество } \\
\text { Махіmum number }\end{array}$ & 30.8 & 31 & 31.5 & 30.5 & 30.1 \\
\hline $\begin{array}{c}\text { Среднее значение } \\
\text { Average number }\end{array}$ & 21.16 & 20.17 & 14.63 & 12.40 & 12.05 \\
\hline
\end{tabular}

Таблица $2^{3}$.

Количество блоков ЦИ, в которых происходит увеличение НОМСЧ при пересохранении с

\begin{tabular}{|c|c|c|c|c|c|}
\hline $\begin{array}{c}\text { Формат ЦИ } \\
\text { Image format }\end{array}$ & TIF & $\begin{array}{c}\text { JPEG } \\
(Q F=90)\end{array}$ & $\begin{array}{c}\text { JPEG } \\
(Q F=80)\end{array}$ & $\begin{array}{c}\text { JPEG } \\
(Q F=75)\end{array}$ & $\begin{array}{c}\text { JPEG } \\
(Q F=70)\end{array}$ \\
\hline $\begin{array}{c}\text { Минимальное количество } \\
\text { Minimal number }\end{array}$ & 56 & 35 & 5.2 & 3 & 2.2 \\
\hline $\begin{array}{c}\text { Максимальное количество } \\
\text { Махітum number }\end{array}$ & 100 & 100 & 100 & 100 & 99.9 \\
\hline Среднее значение & 97.71 & 95.13 & 78.19 & 69.63 & 65.46 \\
\hline
\end{tabular}


2,3 Appendix 1

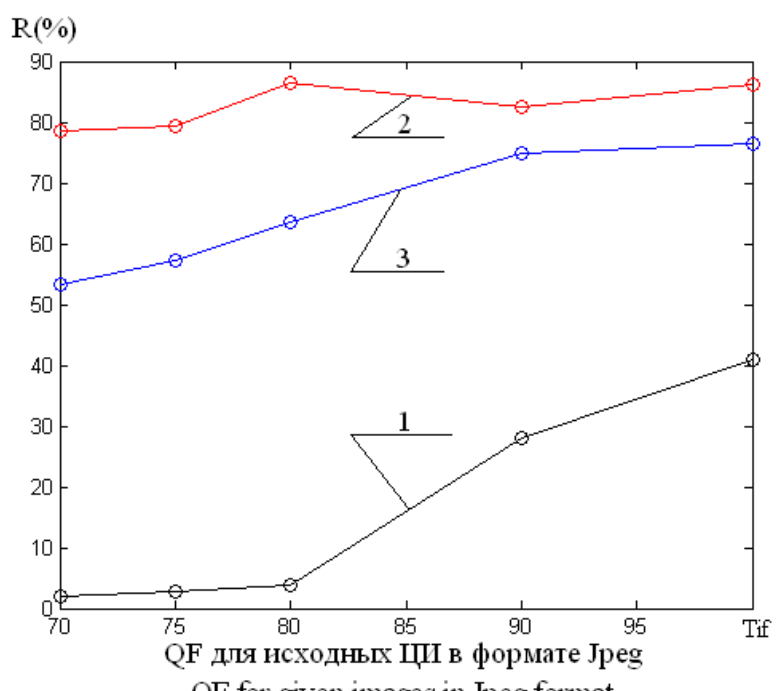

$\mathrm{QF}$ for given images in Jpeg format

Рис. 2. Графики зависимости $R$ - значения разности между количествами блоков ЦИ, для которых увеличилась НОМСЧ при пересохранении с $Q F=1$ и $Q F=100$, от КК исходного изображения в ФсП:

1 - минимальное; 2 - максимальное, 3 - среднее значение по эксперименту. ${ }^{4}$
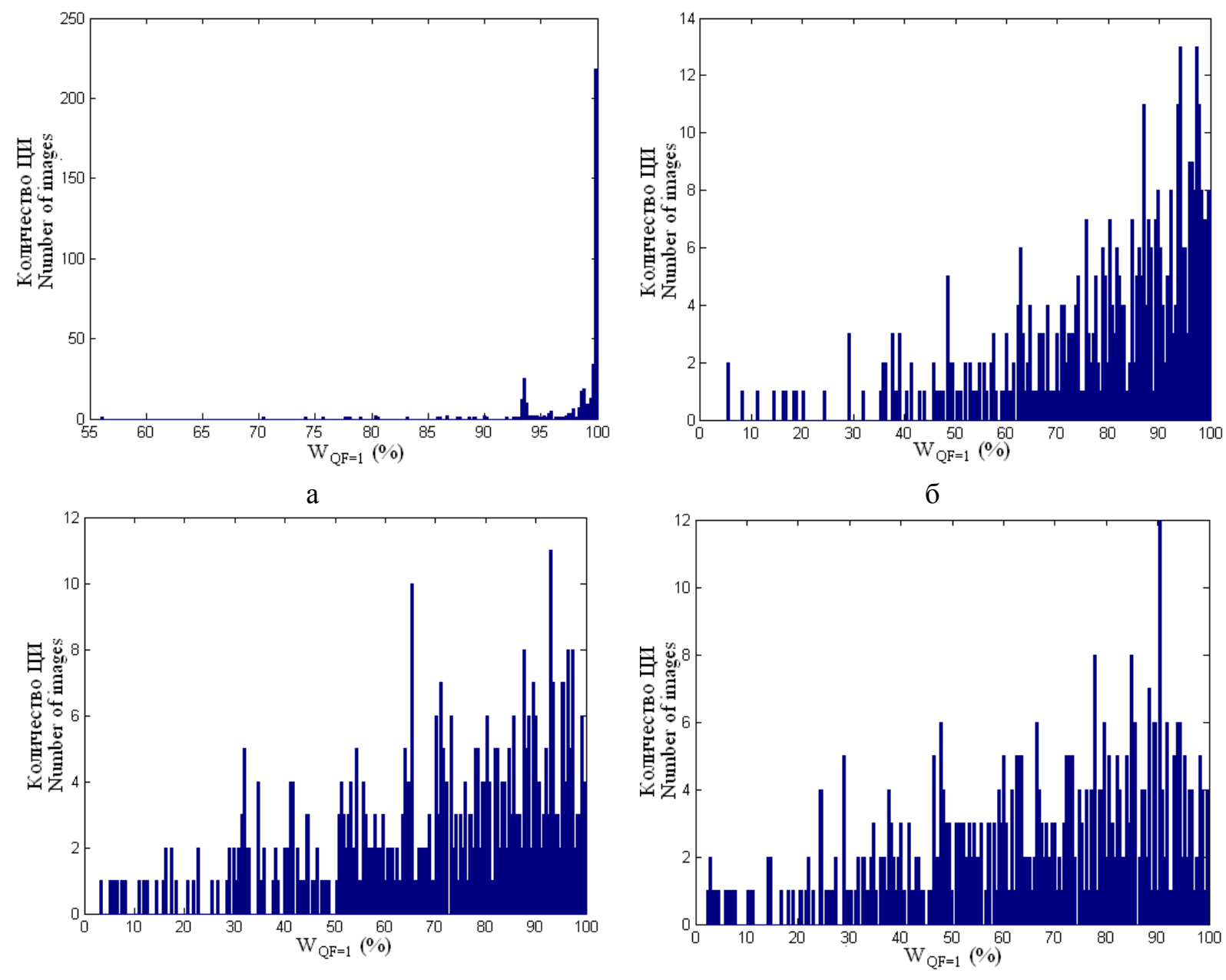

B

$\Gamma$ 
Рис. 3. Гистограммы значений $W_{Q F=1}$ - количества блоков ЦИ, для которых увеличилась НОМСЧ при пересохранении изображения в формат JPEG с $Q F=1$, если исходное ЦИ: а - в формате ТIF; б - в формате JPEG $(Q F=80)$; в - в формате JPEG $(Q F=75) ; \Gamma-$ в формате JPEG $(Q F=70)^{5}$

4,5 Appendix 1

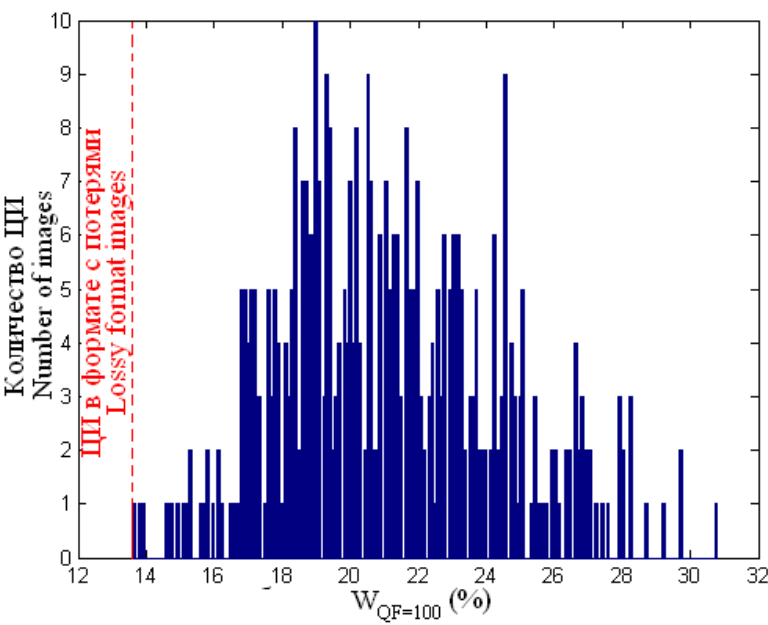

a

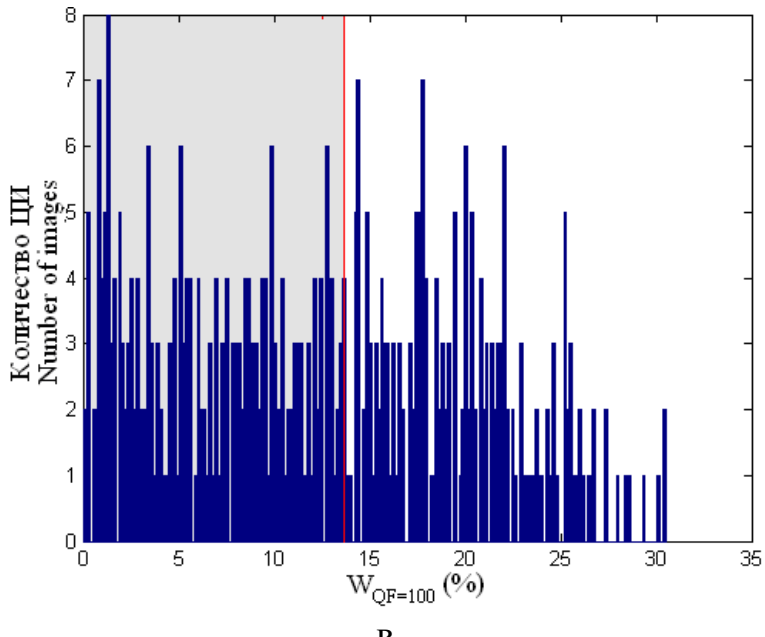

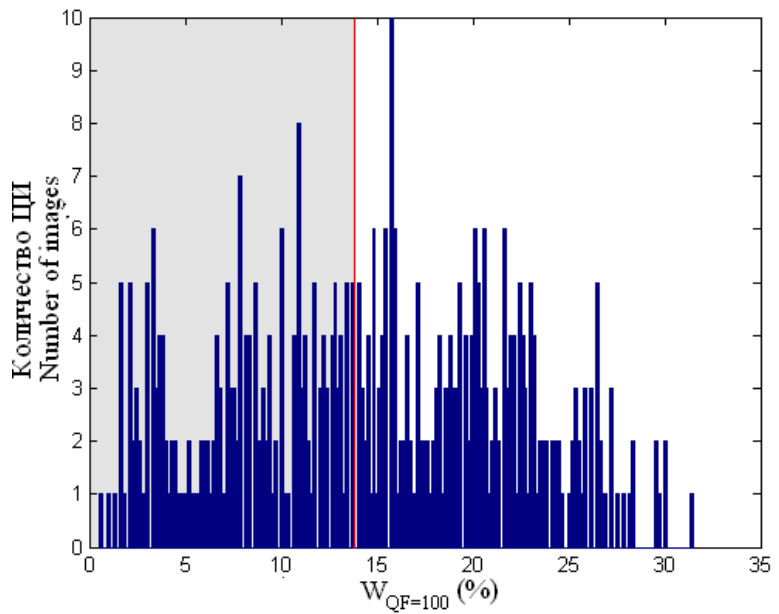

б

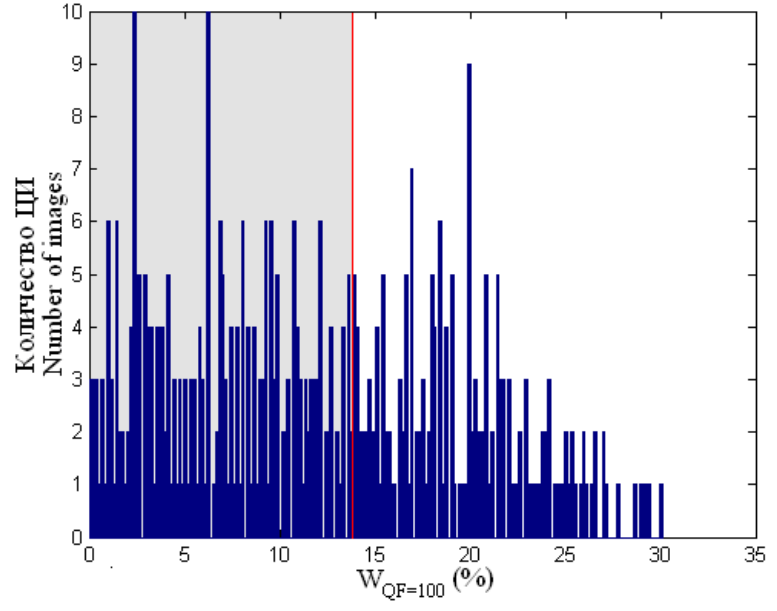

$\Gamma$

Рис. 4. Гистограммы значений $W_{Q F=100}$ - количества блоков ЦИ, для которых увеличилась НОМСЧ при пересохранении изображения в формат JPEG с $Q F=100$, если исходное ЦИ: а - в формате TIF; б - в формате JPEG $(Q F=80)$; в - в формате JPEG $(Q F=75)$; $\Gamma$ - в формате JPEG $(Q F=70)^{6}$

Поэтому предложенное ниже усовершенствование, основанное на учете (7), (11), (12), ожидаемо принесет выигрыш во времени экспертизы не для любого ЦИ, а количество ЦИ, для которых такой выигрыш будет иметь место, будет уменьшаться с ростом КК первичного сжатия.

Метод отделения изображений в различных форматах, его алгоритмическая реализация

Пусть $F-$ матрица ЦИ, которое подвергается экспертизе и формально сохранено в ФбП. Основные шаги метода отделения оригинального ЦИ в ФбП от такого, которое было пересохраненным в ФбП из ФсП, $Q F \in J P$, следующие.

Шаг 1. Пересохранить ЦИ с матрицей $F$ в ФсП с КК $Q F_{\max }(6)$. Матрица полученного изображения $-F_{\max }$.

Шаг 2. Разбить $F_{\max }$ на непересекающиеся $l \times l$-блоки стандартным ${ }^{6}$ Appendix $1 \quad$ образом.

Шаг 3. Определить $y\left(Q F_{\max }\right)$ - количество $l \times l$-блоков ЦИ, для 
которых увеличилась НОМСЧ в результате пересохранения изображения в ФсП с $Q F_{\max }$.

Waг 4.

Если $y\left(Q F_{\text {max }}\right)<T_{1}$, где $T_{1}$ - параметр,

то исследуемое ЦИ первоначально хранилось в ФсП, иначе

4.1. Пересохранить ЦИ с матрицей $F$ в ФсП с КК $Q F_{\min }$ (4). Матрица полученного ЦИ $-F_{\min }$.

4.2. Разбить $F_{\min }$ на $l \times l$-блоки стандартным образом.

4.3. Определить $y\left(Q F_{\min }\right)$.

Если $y\left(Q F_{\min }\right)<T_{2}$, где $T_{2}$ - параметр,

то исследуемое ЦИ первоначально хранилось в ФсП,

иначе
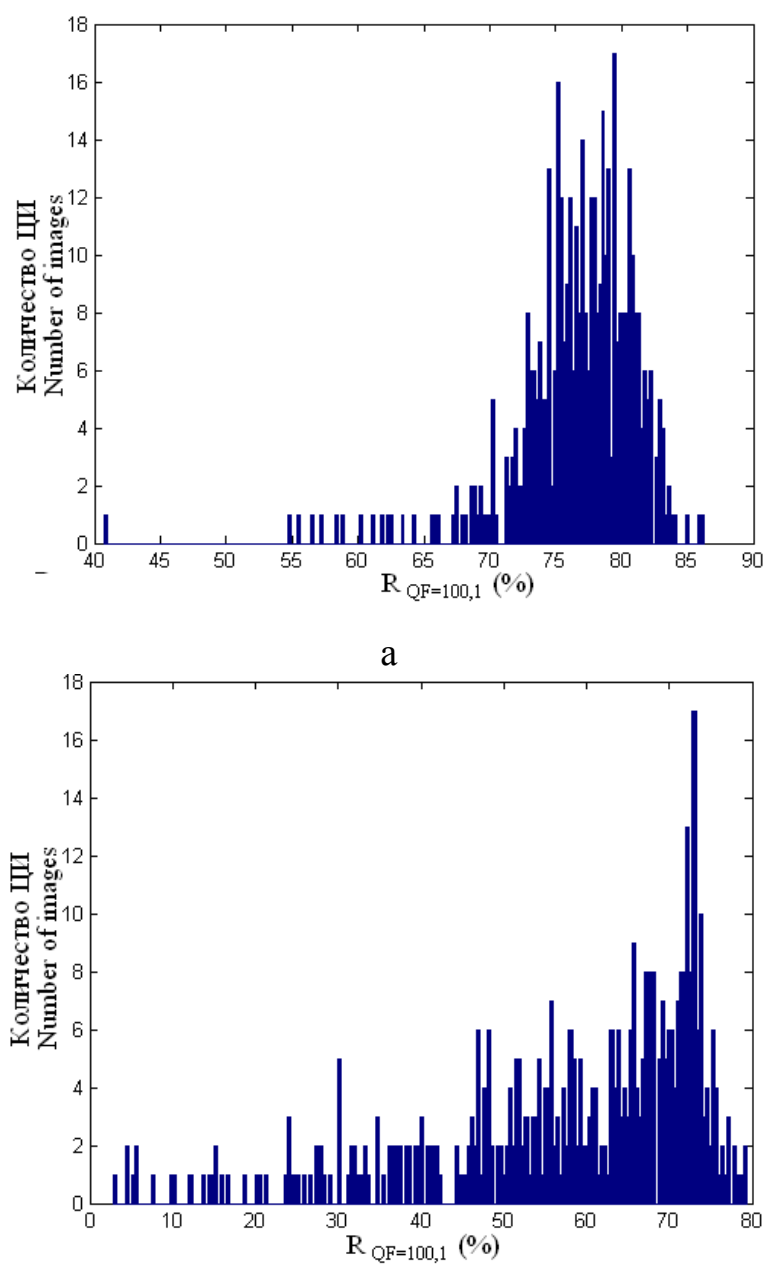

если $y\left(Q F_{\min }\right)-y\left(Q F_{\max }\right)<T_{3}$, где $T_{3}$ - параметр,

то исследуемое ЦИ первоначально хранилось в ФсП,

иначе

построить для ЦИ с матрицей $F$ и исследовать свойства $y(Q F)$, используя $l \times l$-блоки матриц ЦИ, в соответствии с методом, предложенным в [30], пересохраняя ЦИ с матрицей $F$ в ФсП с каждым $Q F \in J P$ (кроме $\left.Q F=Q F_{\max }, \quad Q F=Q F_{\min }\right) . \quad \mathrm{C}$ помощью установленных свойств $y(Q F)$ сделать вывод о реальном формате ЦИ с матрицей $F[30]$.

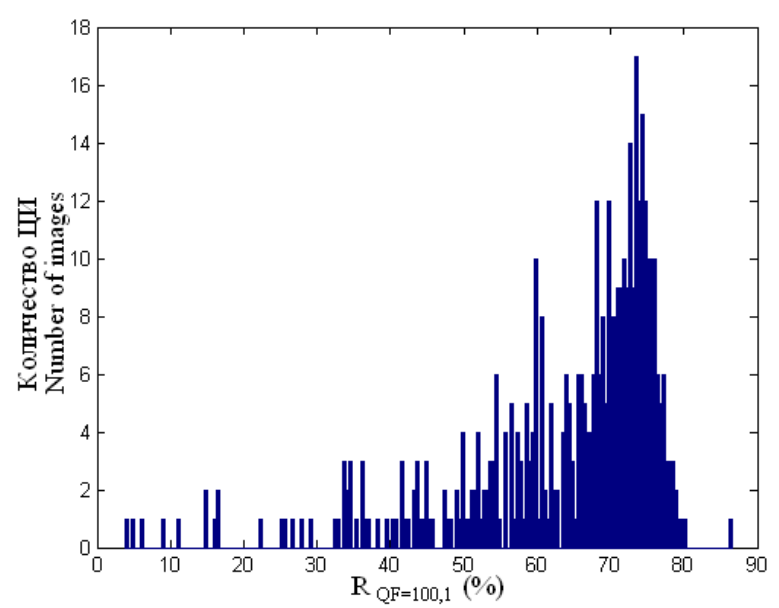

б

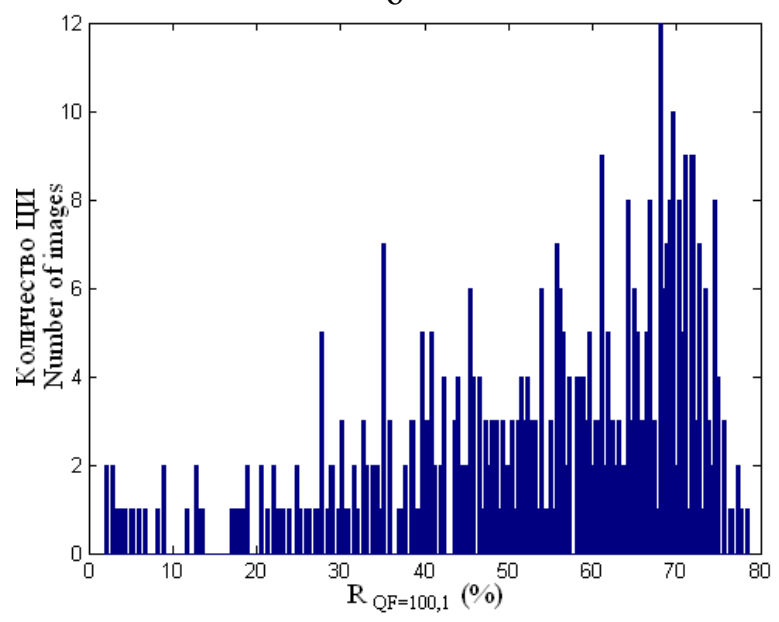

$\Gamma$

Рис. 5. Гистограммы значений $R_{Q F=100,1}$ - разности количества блоков ЦИ, для которых увеличилась НОМСЧ при пересохранении изображения в формат JPEG с $Q F=1$ и $Q F=100$, если исходное ЦИ: а - в формате ТIF; б - в формате JPEG $(Q F=80)$; в - в формате JPEG $(Q F=75)$; $\Gamma-$ в формате JPEG $(Q F=70){ }^{7}$

Разработка алгоритмической реализации распространенного ФсП - JPEG, для которого метода проводилась для наиболее 
$J R=\{1, \ldots, 100\}$. В ходе экспертизы матрица ЦИ разбивалась на $l \times l$-блоки, где $l=4$ [30].

Для определения параметров $T_{1}, T_{2}, T_{3}$ проведен вычислительный эксперимент, в котором были задействованы множества ЦИ:

- $\quad M_{T i f}^{1(400)}, M_{T i f}^{1(600)}, M_{T i f}^{1(800)}$ ЦИ в формате

${ }^{7}$ Appendix 1

TIF (размер ЦИ, которые входят в множества, $400 \times 400$,

$600 \times 600$,

$800 \times 800$ соответственно) из базы [32],

$\left|M_{\text {Tif }}^{1(400)}\right|=\left|M_{\text {Tif }}^{1(600)}\right|=\left|M_{\text {Tif }}^{1(800)}\right|=300$;

- $\quad M_{\text {Tif }}^{2(400)}, M_{\text {Tif }}^{2(600)}, M_{\text {Tif }}^{2(800)}$ ЦИ в формате

TIF (размер ЦИ, которые входят в множества, - 400×400, 600×600, 800×800 соответственно) из базы [33], $\left|M_{\text {Tif }}^{1(400)}\right|=\left|M_{\text {Tif }}^{1(600)}\right|=\left|M_{\text {Tif }}^{1(800)}\right|=150$;

- $M_{J p e g, i}^{1(n)}, \quad i \in\{60,65,70,75,80,85,90\}$, полученных пересохранением ЦИ из $M_{T i f}^{1(n)}$, $n \in\{400,600,800\}$, в формат JPEG с $Q F=i$;

- $M_{\text {Jpeg }, i}^{2(n)}, \quad i \in\{60,65,70,75,80,85,90\}$, полученных пересохранением ЦИ из $M_{T i f}^{2(n)}$, $n \in\{400,600,800\}$, в формат JPEG с $Q F=i$,

т.е. 1350 ЦИ в ФбП (ТIF) и 9450 соответствующих ЦИ в ФсП (JPEG).
В результате эксперимента установлено: $T_{1}=13.5, T_{2}=75.0, T_{3}=55.0$.

\section{III. АНАЛИЗ И РЕЗУЛЬТАТЫ ИССЛЕДОВАНИЯ}

Первый этап вычислительного эксперимента для тестирования алгоритмической реализации разработанного метода использовал: $M_{T i f}^{1(400)}, \quad M_{T i f}^{2(400)}$, $M_{J p e g, i}^{1(400)}, M_{J p e g, i}^{2(400)}, \quad i \in\{60,65,70,75,80,85,90\} . \mathrm{B}$ результате эксперимента установлено, что модифицированный метод позволил уменьшить время экспертизы для 51.2\% ЦИ, которые первоначально хранились в формате JPEG. Для разных значений $Q F$ процент выигрыша в количестве ЦИ был разный. Из выше изложенного понятно, что этот выигрыш будет тем большим, чем меньше $Q F$. Результаты приведены в табл. 3 .

Второй этап эксперимента был посвящен исследованию особенностей работы алгоритма в зависимости от размера ЦИ. На этом этапе использовались: $M_{\text {Tif }}^{1(n)}, \quad M_{J p e g, i}^{1(n)}$, $i \in\{60,65,70,75,80,85,90\}, \quad n \in\{400,600,800\}$. Результаты приведены в табл. 4.

Количество ЦИ, которые первоначально хранились в ФсП, для которых уменьшилось время экспертизы при использовании разработанного алгоритма в сравнении с [30] (\%)

Коэффициент качества QF, который использовался при получении оригинального ЦИ в ФсП Quality factor QF used to obtain the original image in the lossy format

\begin{tabular}{|c|c|c|c|c|c|c|}
\hline$Q F=60$ & $Q F=65$ & $Q F=70$ & $Q F=75$ & $Q F=80$ & $Q F=85$ & $Q F=90$ \\
\hline 71.4 & 70.2 & 65.0 & 60.1 & 50.6 & 32.5 & 10.1 \\
\hline
\end{tabular}

Таблица 4 .

Количество ЦИ, которые первоначально хранились в ФсП, для которых уменьшилось время экспертизы при использовании разработанного алгоритма в сравнении с [30], в зависимости от

\begin{tabular}{|c|c|c|c|c|c|c|c|}
\hline \multicolumn{8}{|c|}{ размера исследуемого ЦИ (\%) } \\
\hline${ }_{n} \quad Q F$ & 60 & 65 & 70 & 75 & 80 & 85 & 90 \\
\hline 400 & 72.7 & 71.0 & 67.0 & 62.7 & 52.3 & 34.0 & 11.0 \\
\hline 600 & 74.7 & 72.3 & 70.0 & 65.3 & 52.7 & 38.0 & 8.7 \\
\hline 800 & 76.3 & 73.7 & 69.7 & 67.3 & 53.3 & 37.3 & 5.3 \\
\hline
\end{tabular}

Значение количества ЦИ, которые первоначально хранились в ФсП, для которых уменьшилось время экспертизы благодаря модификации метода, для каждого $Q F$ практически не зависят от размера ЦИ (табл. 4). Действительно, это количество очевидно определяется характеристиками формата хранения - коэффициентом качества и, как следствие, элементами использованной матрицы квантования, которые не меняются при изменении размера ЦИ.

Вычислим для $Q F \in\{60,65,70,75,80,85,90\}$ среднее значение $S$ количества ЦИ, которые 
первоначально хранились в ФсП, для которых уменьшилось время экспертизы в модифицированном методе, используя для этого табл. 4. График $S(Q F) \quad$ (рис. 6) демонстрирует, что при $Q F<81$ больше, чем для половины ЦИ, которые первоначально хранились в JPEG, наблюдается уменьшения времени экспертизы благодаря учету (7), (11), (12). Показатели уменьшения времени экспертизы ЦИ представлены в табл. 5 (параметры компьютера: Intel Core i73632QM (2.2 ГГц) RAM 4 ГБ). Выигрыш во времени возрастает вместе с ростом размера ЦИ и для $n=800$ превышает 56 раз.

Для оценки эффективности предложенного алгоритма использовались показатели: ошибки I, II рода, АСC (1).

Ошибки I и II рода определялись с использованием следующих множеств: $M_{\text {Tif }}^{1(n)}$,
$M_{\text {Tif }}^{2(n)}, \quad M_{\text {Jpeg,i }}^{1(n)}, \quad M_{\text {Jpeg }, i}^{2(n)}, \quad n \in\{400,600,800\}$, $i \in\{60,65,70,75,80,85,90\}, \quad$ которые в совокупности содержат 10800 ЦИ. Ошибки I рода зафиксированы не были, II рода увеличились в сравнении с [30] на $0.9 \%$ и составили $3.6 \%$. Результаты сравнительной оценки эффективности представлены в табл. 6. Хотя разработанный алгоритм несколько уступает своему прототипу, но также не дает ошибок I рода и значительно уменьшает время вычислений для больше чем половина ЦИ в ФсП. Таким образом, эффективность разработанного алгоритма не зависит от КК сжатия, с которым первоначально было получено ЦИ с потерями, что дает разработанному алгоритма преимущества перед методом [26], хотя значение АСC для них одинаковые.

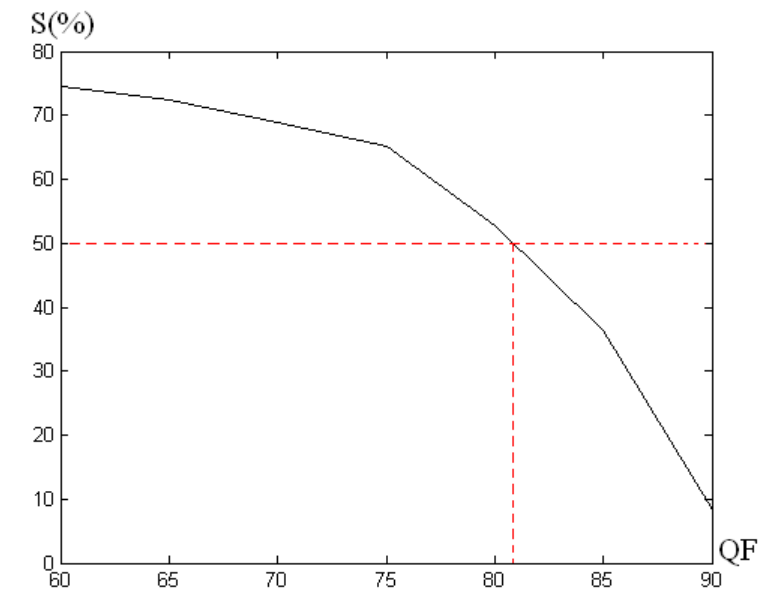

Рис. 6. Зависимость среднего значения количества ЦИ, которые первоначально хранились в ФсП, для которых уменьшилось время экспертизы при использовании разработанного алгоритма в сравнении с [30], от КК первоначального сжатия (\%). ${ }^{10}$

Таблица $5^{11}$. Среднее значение времени работы алгоритмических реализаций усовершенствованного метода и исходного метода [30]

\begin{tabular}{|c|c|c|c|}
\hline $\begin{array}{c}\text { Размер ЦИ } \\
\text { Image size }\end{array}$ & $\begin{array}{c}\text { Усовершенствованный } \\
\text { метод, (c) } \\
\text { Developed method, (s) }\end{array}$ & $\begin{array}{c}\text { Метод [30], (c) } \\
\text { Method [30], (s) }\end{array}$ & $\begin{array}{c}\text { Выигрыш во времени } \\
\text { (раз) } \\
\text { Time gain (times) }\end{array}$ \\
\hline $400 \times 400$ & 1.17 & 45.46 & 38.9 \\
\hline $600 \times 600$ & 1.91 & 102.21 & 53.5 \\
\hline $800 \times 800$ & 3.43 & 194.66 & 56.8 \\
\hline
\end{tabular}

Таблица $6^{12}$.

Сравнение эффективности разработанного алгоритма с современными аналогами

\begin{tabular}{|c|c|c|c|c|c|c|}
\hline Aлгоритмы & $\begin{array}{c}{[24](2007)} \\
\text { Algorithms }\end{array}$ & $\begin{array}{c}{[26](2008)} \\
\text { (в условиях } \\
(\text { when } \mathrm{QF}=75)\end{array}$ & $\begin{array}{c}{[23]} \\
\text { (в условиях } \\
Q F=75) \\
\text { (when QF=75) }\end{array}$ & $\begin{array}{c}{[27]} \\
(2016)\end{array}$ & $\begin{array}{c}{[30]} \\
(2019)\end{array}$ & $\begin{array}{c}\text { Разработанный } \\
\text { (2021) } \\
\text { Developed } \\
\text { (2021) }\end{array}$ \\
\hline ACC & 0.7 & 0.98 & 0.96 & $0.96 / 0.95$ & 0.99 & $\mathbf{0 . 9 8}$ \\
\hline
\end{tabular}


Теоретический базис предложенного метода дает возможность его использования для выявления нарушений целостности ЦИ в результате незначительных возмущающих воздействий, что может быть проиллюстрировано на примере двух ЦИ, сохраненных

10,11,12 Appendix 1 первоначально формате JPEG. В качестве возмущающих воздействий использовались: LSB з ПСПК $=0.01,0.05,0.1$ бит/пиксель; гауссовский шум с $D=0.00001$; мультипликативный шум с $D=0.0001$. Нарушения целостности в каждом случае были выявлены, при этом на экспертизу одного из ЦИ было затрачено 19 с., другого 1183 с., т.е. временной выигрыш здесь составил более 62 раз.

\section{ВЫВОДЫ}

В работе проведено усовершенствование метода [30] отделения оригинального ЦИ в ФбП от такого, которое было пересохранено в ФбП из ФсП. Предложенное усовершенствование привело к значительному уменьшению вычислительной сложности при экспертизе больше чем 50\% ЦИ, которые первоначально хранились в ФсП. Уменьшение вычислительной сложности получено благодаря установленным и использованным дополнительным свойствам функции $y(Q F)$, которая отражает зависимость количества блоков ЦИ, в которых увеличивается НОМСЧ при пересохранении в ФсП, от КК $Q F$, в значениях аргумента $Q F=Q F_{\max }$, $Q F=Q F_{\min } . \quad$ Установленные свойства $y\left(Q F_{\max }\right), \quad y\left(Q F_{\min }\right) \quad$ дали возможность уменьшить количество пересохранений исследуемого ЦИ в ФсП при его экспертизе до 1-2 раз, что привело к уменьшению времени экспертизы в 38-56 раз в сравнении с алгоритмом-прототипом. При этом предложенный алгоритм имеет высокую эффективность отделения ЦИ в разных (с/без потерь) форматах хранения: ошибки I рода отсутствуют, II рода составили $3.6 \%$; $\mathrm{ACC}=0.98$, что превышает все современные аналоги, кроме алгоритма-прототипа; АCC не зависит от КК ЦИ, которое первоначально хранилось в ФсП, что дает преимущество разработанному алгоритму перед аналогами
$[24,26]$, даже с учетом того, что АCC для разработанного и предложенного в [26] одинаковые.

Значительным преимуществом разработанного метода и его алгоритма перед аналогами [23-27] является то, что теоретический базис дает возможность для их использования не только для отделения ЦИ в ФбП от ЦИ в ФсП, а в целом при экспертизе целостности ЦИ, нарушение которой происходит в результате незначительного возмущающего воздействия, в частности для стеганоанализа в условиях малой пропускной способности скрытого канала связи.

\section{APPENDIX 1 (ПРИЛОЖЕНИЕ 1)}

${ }^{1}$ Fig. 1. Graph of function $y(Q F)$ and $\bar{y}(Q F)$ for corresponding images: $1-y(Q F)$ for image in TIFF format; 2, $3-y(Q F)$ for image in JPEG $(Q F=85)$ and $\bar{y}(Q F)$ respectively; $4,5-y(Q F)$ for image in JPEG $(Q F=75)$ and $\bar{y}(Q F)$ respectively; 6, $7-y(Q F)$ for image in JPEG $(Q F=65)$ and $\bar{y}(Q F)$ respectively

${ }^{2}$ Table 1. The number of image blocks in which the normalized gap of maximum singular value increases when image was re-saved with losses with $Q F=100$ (\%).

${ }^{3}$ Table 2. The number of image blocks in which the normalized gap of maximum singular value increases when image was re-saved with losses with $Q F=1(\%)$.

${ }^{4}$ Fig. 2. Graphs of the values of the $R$ - difference between the number of image blocks, for which the normalized gap of maximum singular value has increased when re-saving image with $Q F=1$ and $Q F=100$, versus the quality factor of the given image in a lossy format: 1 - the minimum value according to the experiment; 2 - the maximum value for the experiment, 3 - the average value for the experiment

${ }^{5}$ Fig. 3. Histograms of $W_{Q F=1}$ - number of image blocks, for which the normalized gap of maximum singular value has increased when the image was resaved into the Jpeg format with $Q F=1$, if the given image is: (a) - in the TIFF; (b) - in JPEG ( $Q F=80$ ); (c) - in JPEG $(Q F=75)$; (d) - in JPEG $(Q F=70)$

${ }^{6}$ Fig. 4. Histograms of $W_{Q F=100}$ number of image blocks, for which the normalized gap of maximum singular value has increased when the image was resaved into the Jpeg format with $\mathrm{QF}=100$, if the given image is: (a) - in the TIFF; (b) - in JPEG ( $Q F=80$ ); (c) - in JPEG $(Q F=75)$; (d) - in JPEG $(Q F=70)$

${ }^{7}$ Fig. 5. Histograms which represent $R_{Q F=100,1}$ - the values of the difference between the number of image blocks, for which the normalized gap of maximum singular value increased when the image was re-saved 
into the Jpeg format with $Q F=1$ and $Q F=100(\%)$, if the given image is: (a) - in the TIFF; (b) - in JPEG $(Q F=80) ;(\mathrm{c})-$ in JPEG $(Q F=75) ;(\mathrm{d})-$ in JPEG $(Q F=70)$

${ }^{8}$ Table 3. The number of images that were initially stored in a lossy format for which the examination time was reduced by the developed algorithm compared to [30] (\%)

${ }^{9}$ Table 4. The number of images which were initially stored in a lossy format, for which the examination time was reduced when using the developed algorithm in comparison with [30], depending on the size of the studied image

${ }^{10}$ Fig. 6. The average value of the number of images, which were initially stored in the format with losses, for which the examination time was reduced using the developed algorithm in comparison with [30], versus the quality factor of the initial compression (\%)

${ }^{11}$ Table 5. The average value of the running time of algorithmic implementations of the developed method and the method [30], taken as a basis

${ }^{12}$ Table 6. Comparison of the effectiveness of the developed algorithm with modern analogues

\section{Литература (References)}

[1] Miller K.W., Voas J. Information Integrity and IT Professionals' Integrity, Intertwined. IT Professional, 2008, 10, 35-40. doi:10.1109/MITP.2008.124.

[2] Geisler E., Prabhaker P., Nayar M. Information Integrity: an Emerging Field and the State of Knowledge. Proc. of Portland International Conference on Management of Engineering and Technology Management for Reshaping the World (PICMET'03), Portland, USA, 24-24 July 2003; IEEE, 2003. doi:10.1109/PICMET.2003.1222797.

[3] Flowerday S., von Solms R. What Constitutes Information Integrity? SA Journal of Information Management, 2007, 9, a201. doi:10.4102/sajim.v9i4.201.

[4] Limaye R.S. The Importance of Information Integrity, Security, Networking and Data Protection. International Journal of Innovations in Engineering and Technology, 2013, 2, 274281 .

[5] Solovyov V.I., Rybalskiy O.V., Zhuravel V.V. Substantiating the Fundamental Fitness of Deep Learning Neural Networks for Construction of a Phonogram Digital Processing Detection System. Cybernetics and Systems Analysis, 2020, 56, 326-330. doi:10.1007/s10559-020-00249-2.

[6] Joglekar N.P., Chatur P.N. A Compressive Survey on Active and Passive Methods for Image Forgery Detection. International Journal of Engineering and Computer Science, 2015, 4, 10187-10190.

[7] Shwetha B., Sathyanarayana S.V. Digital Image Forgery Detection Techniques: a Survey.
ACCENTS Transactions on Information Security, 2017, 2, 22-31. doi:10.19101/TIS.2017.25003.

[8] Duan X., Liu N., Gou M., Wang W., Qin C. SteganoCNN: Image Steganography with Generalization Ability Based on Convolutional Neural Network. Entropy, 2020, 22, 1140. doi:10.3390/e22101140

[9] Jarusek R., Volna E., Kotyrba M. Photomontage Detection Using Steganography Technique Based on a Neural Network. Neural Networks, 2019, 116, 150-165. doi:10.1016/j.neunet.2019.03.015.

[10]Zheng L., Zhang Y., Thing V.L.L. A Survey on Image Tampering and its Detection in RealWorld Photos. Journal of Visual Communication and Image Representation, 2019, 58, 380-399. doi:10.1016/j.jvcir.2018.12.022.

[11] Akram Hatem Saber, Mohd Ayyub Khan, Basim Galeb Mejbel. A Survey on Image Forgery Detection Using Different Forensic Approaches. Advances in Science, Technology and Engineering Systems Journal, 2020, 5(3), 361370. doi: 10.25046/aj050347.

[12] Yahya A. Steganography Techniques for Digital Images. Berlin, Springer, 2019. 138 p.

[13] Varalakshmi R. Digital Steganography for Preventing Cybercrime Using Artificial Intelligence Technology. Journal of Critical Reviews, 2020, 7, 749-753. doi:10.31838/jcr.07.06.132.

[14] Agranovsky A.V., Balakin A.V., Gribunin V.G., Sapozhnikov S.A. Steganografiya, Tsifrovye Vodyanye Znaki, Steganoanaliz [Steganography, Digital Watermarks, and Steganalysis]. Moscow, 2009. (In Russian).

[15] Veena S.T., Arivazhagan S. Universal Secret Payload Location Identification in Spatial LSBstego Images. Annals of Telecommunications, 2019, 74, 273-286. doi:10.1007/S12243-018-0676-X.

[16] Amarendra K. e.a. Image Steganography Using LSB. International Journal of Scientific \& Technology Research, 2019, 8, 906-909.

[17] Lukin V. e.a. Lossy Compression of Multichannel Remote Sensing Images with Quality Control. Remote Sens., $\quad 2020, \quad 3840$. https://doi.org/10.3390/rs12223840

[18] Li B., He J., Huang J., Shi Y. A Survey on Image Steganography and Steganalysis. Journal of Information Hiding and Multimedia Signal Processing, 2011, 2, 142-172.

[19] Karampidis K., Kavallieratou E., Papadourakis G. A Review of Image Steganalysis Techniques for Digital Forensics. Journal of Information Security and Applications, $\quad 2018, \quad 40, \quad 217-235$. doi:10.1016/j.jisa.2018.04.005.

[20]Liu F., Zhou X., Yan X., Lu Y., Wang S. Image Steganalysis via Diverse Filters and Squeeze-andExcitation Convolutional Neural Network. $\begin{array}{llll}\text { Mathematics, } & 2021, & 9, & 189 .\end{array}$ https://doi.org/10.3390/math9020189 
[21] Salomon M., Couturier R., Guyeux C., Couchot J.-F., Bahi J.M. Steganalysis via a Convolutional Neural Network Using Large Convolution Filters for Embedding Process with Same Stego Key: A Deep Learning Approach for Telemedicine. European Research in Telemedicine, 2017, 6, 79-92. doi:10.1016/j.eurtel.2017.06.001.

[22] Wang Z., Chen M., Yang Y., Lei M., Dong Z. Joint Multi-Domain Feature Learning for Image Steganalysis Based on CNN. EURASIP Journal on Image and Video Processing, 2020, 28. doi:10.1186/s13640-020-00513-7.

[23] Akhmametieva A. Method of Detection the Fact of Compression in Digital Images as an Integral Part of Steganalysis. Informatics and Mathematical Methods in Simulation, 2016, 6, 357-364.

[24] Tjoa S., Lin W.S., Zhao H.V., Liu K.J.R. Block Size Forensic Analysis in Digital Images. Proc. of the 2007 IEEE International Conference on Acoustics, Speech and Signal Processing (ICASSP'07), Honolulu, USA, 15-20 April 2007. IEEE, 2007. pp. I-633-I-636.

[25] Geetha S., Sindhu S., Kamaraj N. Close Color Pair Signature Ensemble Adaptive Threshold Based Steganalysis for LSB Embedding in Digital Images. Transactions on Data Privacy, 2009, 1, 140-161.

[26] Luo W., Huang J., Qiu G. A Novel Method for Block Size Forensics Based on Morphological Operations. In: Kim H.J., Katzenbeisser S., Ho A.T.S. (eds) Digital Watermarking. IWDW 2008. Lecture Notes in Computer Science, vol 5450. Springer, Berlin,

\section{Сведения об авторах.}

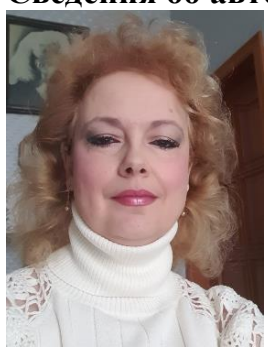

Кобозева Алла Анатольевна. Национальный университет «Одесская политехника». Кафедра кибербезопасности и программного обеспечения, доктор технических наук, профессор. Область научных интересов: информационная безопасность, в частности, стеганография, E-mail: alla_kobozeva@ukr.net

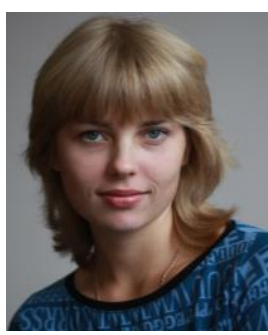

Кушниренко Наталия Игоревна. Национальный университет «Одесская политехника». Кафедра кибербезопасности и программного обеспечения, кандидат технических наук, доцент. Область научных интересов: информационная безопасность.

E-mail: kushnirenko@op.edu.ua
Heidelberg. https://doi.org/10.1007/978-3-642-044380_20

[27] Bobok I.I. Development of the Method for Separating Digital Image Stored in a Lossless Format from Stored with Losses. Modern Special Technics, 2018, 4, 15-24.

[28]Baker K. Singular Value Decomposition Tutorial, March 2005. Available Online: https://datajobs.com/data-science-repo/SVD-Tutorial[Kirk-Baker].pdf (accessed on 20 March 2021).

[29] Demmel J.W. Applied Numerical Linear Algebra. SIAM: Philadelphia, USA, 1997.

[30] Bobok I.I., Kobozeva A.A. Steganalysis Method Efficient for the Hidden Communication Channel with Low Capacity. Radiotechnika, 2019, 198, 19-31. doi:10.30837/rt.2019.3.198.02.

[31] Gonzalez R.C., Woods R.E. Digital Image Processing. Pearson: Upper Saddle River, USA, 2018.

[32] Gloe T., Böhme R. The "Dresden Image Database" for Benchmarking Digital Image $f$

[33] Forensics. Proc. of the 2010 ACM Symposium on Applied Computing (SAC'10), Sierre, Switzerland, 2226 March 2010. Association for Computing Machinery: NY, USA, 2010. pp. 1585-1591.

[34] Hsu Y., Chang S. Detecting Image Splicing using Geometry Invariants and Camera Characteristics Consistency. Proc. of the 2006 IEEE International Conference on Multimedia and Expo, Toronto, Canada, 9-12 July 2006; IEEE, 2006; pp. 549-552. doi:10.1109/ICME.2006.262447

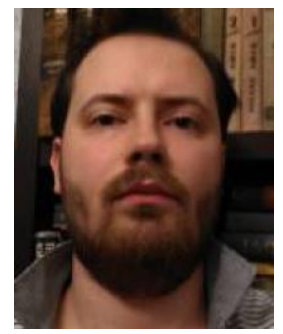

Бобок Иван Игоревич.

Национальный университет «Одесская политехника». Кафедра компьютеризованных систем управления, доктор технических наук, доцент. Область научных интересов: информационная безопасность, безопасность социотехнических систем. E-mail: onu_metal@ukr.net 